\title{
Colangiografía endoscópica: disminuyendo complicaciones
}

\author{
Aurelio López-Colombo* \\ Dirección de Educación e Investigación en Salud, Hospital de Especialidades del Centro Médico Nacional Manuel Ávila Camacho, Instituto \\ Mexicano del Seguro Social, Puebla, Puebla, México
}

\begin{abstract}
Resumen
El empleo de la colangiopancratografía endoscópica va en aumento primordialmente como una herramienta terapéutica y particularmente para el manejo de la colédoco litiasis. Sin embargo, este procedimiento no está exento de riesgos. La pancreatitis es el evento adverso serio más común, por lo que esta revisión se centra en esta complicación. Se contrastan las recomendaciones de las guías actuales con la nueva evidencia. Desafortunadamente los resultados de los estudios que han probado nuevas estrategias aún no han mostrado beneficios claros, por lo que la mejor decisión para el endoscopista es apegarse a las recomendaciones de las guías.
\end{abstract}

Palabras clave: Colangiopancreatografía endoscópica (ERCP). Pancreatitis post-CPRE (PEP). Complicaciones. Guías de práctica clínica.

La colangiopancreatografía endoscópica (CPE o CPRE), fue descrita inicialmente por MaCune en 1968 como un procedimiento para evaluar la vía biliar y el conducto pancreático ${ }^{1}$. A partir de 1974 en que se introdujo la esfinterotomía endoscópica, la CPE se convirtió en un procedimiento diagnóstico y terapéutico para resolver patologías de las vías biliares y el páncreas $^{2}$. El perfeccionamiento de las técnicas de imagen desde el ultrasonido hasta la resonancia magnética han modificado la historia de la CPRE, que evolucionó de ser un procedimiento diagnóstico para convertirse en un procedimiento casi exclusivamente terapéutico. Contrario a lo que se pudiera pensar, el empleo de la CPRE ha ido en aumento en los últimos años primordialmente para el manejo de colédoco litiasis ${ }^{3}$. Esto tiene que ver con el advenimiento de la cirugía laparoscópica y las preferencias de los cirujanos. Un estudio reciente muestra una marcada disminución en el uso de la exploración de vías biliares tanto abierta como laparoscópica 4 .

Sin embargo, este procedimiento no está exento de riesgos. De los eventos adversos serios, no cabe duda que la pancreatitis post CPRE (PEP por sus siglas en inglés: post-ERCP pancreatitis) es el más común, con una incidencia de $9.7 \%$ (IC 95\% 8.6-10.7\%), una mortalidad de $0.7 \%$ y costos que exceden anualmente los 150 millones de dólares en los Estados Unidos ${ }^{5,6}$. Es por esto que el objetivo de esta revisión se concentrará en la prevención de la PEP, que fue uno de los temas del simposio conjunto de la Asociación Mexicana de Endoscopía Gastrointestinal (AMEG) y la American Society of Gastrointestinal Endoscopy (ASGE) en la Semana de Enfermedades Digestivas 2019 (DDW 2019) de los Estados Unidos.

Aunque la PEP es un evento impredecible aún para endoscopistas expertos, se han identificado factores

\section{Correspondencia:}


de riesgo, los cuales vale la pena considerar cuando se planea indicar o realizar una $\mathrm{CPRE}^{6}$. Una selección cuidadosa de los pacientes que serán sometidos a CPRE se considera una de las estrategias más eficaces para evitar PEP. La estratificación de los riesgos de acuerdo las características propias del paciente 0 al procedimiento, debe guiar a la implementación de las posibles intervenciones profilácticas?

Con base en la evidencia disponible, en la actualidad, la mayoría de guías de práctica clínica, recomiendan la administración rutinaria de anti inflamatorios no esteroideos (AINE) antes, durante o después del procedimiento; sobre hidratación con soluciones intravenosas (preferentemente Ringer Lactato); la utilización de guía para la canulación selectiva de la vía biliar; y para los pacientes con alto riesgo de desarrollar PEP, la utilización de prótesis pancreáticas ${ }^{6,8}$. ¿Hay nueva evidencia?

Vale la pena señalar que en el último año se han publicado tres nuevos meta análisis sobre el uso de AINE para la prevención de PEP ${ }^{9-11}$. Los tres artículos tienen varias cosas en común, aunque también algunas diferencias. Las semejanzas que vale le pena destacar es que son estudios de buena calidad metodológica, son meta análisis o revisiones sistemáticas y meta análisis de ensayos clínicos aleatorizados. El objetivo de los tres fue evaluar la eficacia del uso profiláctico de los AINE versus placebo o no tratamiento. El desenlace principal fue la frecuencia de PEP en los diferentes grupos. Y lo más importante es que en los tres artículos se confirmó la eficacia de los AINE para prevenir PEP.

A diferencia de los otros dos estudios, el meta análisis de $\mathrm{He}$, et al. únicamente evaluó la administración de indometacina rectal, la cual demostró ser útil para la prevención de PEP tanto en pacientes de alto riesgo como de riesgo promedio. ${ }^{9}$ Los otros dos meta análisis evaluaron diferentes AINE, vías y momentos de administración. El estudio de Lyu mostró que los AINE disminuyeron significativamente la PEP (RR, $0.61,95 \% \mathrm{Cl}$, $0.52-0.72 ; p<0.00001)$. Con respecto a la vía de administración, este estudio solo mostró eficacia cuando la administración del fármaco fue por vía rectal. La indometacina administrada posterior al procedimiento pareció ser más eficaz que si era administrada previa al procedimiento, contrario a la administración diclofenaco, cuya eficacia fue superior cuando se administró antes del procedimiento ${ }^{10}$. Estos desenlaces secundarios contrastaron con el estudio de Liu en el que no se evidenciaron diferencias entre los diferentes AINE, rutas y momentos de administración ${ }^{11}$.
Sobre el uso de prótesis pancreáticas en pacientes de alto riesgo, además de estudios confirmatorios recientemente se han publicado dos estudios que sugieren que el uso de prótesis mayores de $5 \mathrm{Fr}$ y mayores de $5 \mathrm{~cm}$ (o que alcancen cuerpo o cola), es más eficaz para prevenir PEP que el uso de prótesis de menor calibre o menor longitud ${ }^{12,13}$. Vale la pena señalar que ambos estudios fueron retrospectivos, por lo que se requiere de más estudios, particularmente de ensayos clínicos que confirmen estos hallazgos.

A pesar de las recomendaciones, la tasa de PEP sigue siendo alta, por lo que se tiene la esperanza de que nuevas estrategias disminuyan drásticamente esta complicación. Desafortunadamente la literatura está llena de resultados negativos. Tal es el caso de la administración de un la $\mathrm{N}$-acetil cisteína, un antioxidante que se administró concomitantemente con indometacina pero que resultó no ser superior a la administración de indometacina sola ${ }^{14}$. Un ensayo clínico aleatorizado de 146 pacientes comparó el empleo de medio de contraste isoosmolar (iodixanol) vs el medio de contraste convencional (hiperosmolar) y no encontró diferencias en la tasa de PEP, aunque la frecuencia de casos graves fue mayor en el grupo de contraste hiperosmolar ${ }^{15}$. Otra estrategia que se ha evaluado recientemente es la aplicación tópica (en espray) de epinefrina sobre la pápila durante la CPRE, lo cual no solo no mostró eficacia, sino que aparentemente aumentó el riesgo de desarrollar pancreatitis ${ }^{16,17}$.

Con esta información se puede concluir que la nueva evidencia apoya la vieja evidencia. La evidencia para o contra el uso de nuevas estrategias aún no ofrece resultados claros, por lo que hay que esperar los resultados de más investigación. En este momento la mejor decisión es la de apegarse a las recomendaciones de las guías de práctica clínica.

\section{Bibliografía}

1. McCune WS, Shorb PE, Moscovitz H. Endoscopic cannulation of the ampulla of Vater: a preliminary report. Ann Surg 1968;167:752-6.

2. Kawai K, Akasaka Y, Murakami K, Tada M, Koli Y. Endoscopic sphincterotomy of the ampulla of Vater. Gastrointest Endosc 1974;20:148-51.

3. Moffatt DC, Yu BN, Yei $W$ et al. Trends in utilization of diagnostic and therapeutic endoscopic retrograde cholangiopancreatography (ERCP) in Manitoba: a population based study. Gastrointest Endosc 2014;79:615-22.

4. Wandling MW, HUngness ES, Pavey ES et al. Nationwide assessment of trends in choledocolitiasis management in the United States froma 1998 to 2013. JAMA Surg. 2016;151(12):1125-1130.

5. Kochar B, Akshintala VS, Afghani E et al. Incidence, severity, and mortality of post ERCP pancreatitis: a systematic review by using randomized, controlled trials. Gastrointest Endosc 2015;81:143-149.

6. Chandrasekhara V, Khashab MA, Muthusamy R et al. Adverse events associated with ERCP. Gastrointest Endosc 2017;85(1):33-47.

7. Leerhoy B, Elmunzer BJ. How to Avoid Post-Endoscopic Retrograde Cholangiopancreatography pancreatitis. Gastrointest Endoscopy Clin N Am 2018;28(4) 439-454. 
8. Dumonceau JM, Andriulli A, Elmunzer BJ el al. Prophylaxis of post-ERCP pancreatitis: European Society of Gastrointestinal Endoscopy (ESGE) Guideline - updated June 2014. Endoscopy. 2014 Sep;46(9):799-815. doi: 10.1055/s-0034-1377875

9. He X, Zheng W, Ding $Y$ et al. Rectal Indomethacin Is Protective against Pancreatitis after Endoscopic Retrograde Cholangiopancreatography: Systematic Review and Meta-Analysis. Gastroenterol Res Pract. 2018:9784841. doi: 10.1155/2018/9784841.

10. Lyu $Y$, Cheng $Y$, Wang $B$ et al. What is impact of nonsteroidal anti-inflammatory drugs in the prevention of post-endoscopic retrograde cholangiopancreatography pancreatitis: a meta-analysis of randomized controlled trials. BMC Gastroenterol 2018;18(1):106.

11. Liu L, Li C, Huang Y, Jin H. Nonsteroidal Anti-inflammatory Drugs for Endoscopic Retrograde Cholangiopancreatography Postoperative Pancreatitis Prevention: a Systematic Review and Meta-analysis. J Gastrointest Surg 2018 doi: 10.1007/s11605-018-3967-7.

12. Olsson G, Lübbe J, Arnelo U et al. The impact of prophylactic pancreatic stenting on post-ERCP pancreatitis: A nationwide, register-based study. United European Gastroenterol J. 2017;5(1):111-118.
13. Sugimoto M, Takagi T, Suzuki $R$ et al. Pancreatic stents for the prevention of post-endoscopic retrograde cholangiopancreatography pancreatitis should be inserted up to the pancreatic body or tail. World J Gastroenterol 2018:24(22):2392-2399.

14. Pavel L, Bălan GG, Nicorescu A et al. Split-dose or hybrid nonsteroidal anti-inflammatory drugs and $\mathrm{N}$-acetylcysteine therapy for prevention of post-retrograde cholangiopancreatography pancreatitis. World J Clin $\mathrm{Ca}$ ses 2019; 7(3): 300-310.

15. Ogura T, Imoto A, Okuda A et al. Can lodixanol Prevent Post-Endoscopic Retrograde Cholangiopancreatography Pancreatitis? A Prospective, Randomized, Controlled Trial. Dig Dis. 2019:37(3):255-261.

16. Luo $H$, Wang $X$, Zhang $R$ et al. Rectal Indomethacin and Spraying of Duodenal Papilla With Epinephrine Increases Risk of Pancreatitis Following Endoscopic Retrograde Cholangiopancreatography. Clin Gastroenterol Hepatol. 2018; pii: S1542-3565(18)31209-6. [Epub ahead of print]

17. Kamal A, Akshintala VS, Talukdar R et al. A Randomized Trial of Topical Epinephrine and Rectal Indomethacin for Preventing Post-Endoscopic Retrograde Cholangiopancreatography Pancreatitis in High-Risk Patients. Am J Gastroenterol 2019;114(2):339-347. 